\title{
A Hybrid Systems Approach to Trajectory Tracking Control for Juggling Systems*
}

\author{
Ricardo G. Sanfelice, Andrew R. Teel, and Rodolphe Sepulchre
}

\begin{abstract}
From a hybrid systems point of view, we provide a modeling framework and a trajectory tracking control design methodology for juggling systems. We present the main ideas and concepts in a one degree-of-freedom juggler, which consists of a ball bouncing on an actuated robot. We design a hybrid control strategy that, with only information of the ball's state at impacts, controls the ball to track a reference rhythmic pattern with arbitrary precision. We extend this hybrid control strategy to the case of juggling multiple balls with different rhythmic patterns. Simulation results for juggling of one and three balls with a single actuated robot are presented.
\end{abstract}

\section{INTRODUCTION}

Mechanical systems with impacts are nonsmooth dynamical systems with trajectories that have intervals of continuity (flow) and points of discontinuity (jumps). Several frameworks for modeling these systems have been proposed in the literature, including Poincaré map modeling [3], [14], [15], [12], dynamical systems with unilateral constraints [1], [15], [2], and measure differential inclusions [7].

Following the framework in [4] (see also [5], [10]), we model mechanical systems with impacts as hybrid dynamical systems where flows are given by a differential equation/inclusion and jumps by a difference equation/inclusion, on specific subsets of the state space. In this paper, we focus our attention on a particular class of mechanical systems with impacts: juggling systems. Juggling systems consist of a plant, given by one or many objects, controlled at impacts by an actuated robot [3], [6].

The problem of stabilization of juggling systems to rhythmic patterns has received great attention from the engineering and neuroscience community because of its relevance in robotics and nature. A widely used benchmark juggling system for this type of task is the one degree-of-freedom juggler, which consists of a ball bouncing vertically on an actuated one degree-of-freedom robot. Notable references on this topic include the feedback control strategies in [15], [12], [9] and the open-loop strategies in [11], [8] for phaselock stabilization to rhythmic patterns. Our novel modeling framework for juggling systems, which permits the combination of both continuous-time and discrete-time features in

R.G. Sanfelice: Laboratory for Information and Decision Systems, Massachusetts Institute of Technology, MA 02139, sricardo@mit.edu (research performed at the Department of Electrical and Computer Engineering, University of California, Santa Barbara); A.R. Teel: Department of Electrical and Computer Engineering, University of California, Santa Barbara, CA 93106-9560,teel@ece.ucsb.edu; R. Sepulchre: Department of Electrical and Computer Science (Montefiore Institute), Université de Liège, 4000 Liège, Belgium, R.Sepulchre@ulg.ac.be.

${ }^{*}$ Research partially supported by the National Science Foundation under Grant no. CCR-0311084 and Grant no. ECS-0622253, and by the Air Force Office of Scientific Research under Grant no. FA9550-06-1-0134. the control algorithm and uses an extended time domain for the system solutions (called hybrid time domain), allow us to accomplish trajectory tracking tasks as well. We propose a hybrid control strategy for the one degree-of-freedom juggler which, by relying only on measurements of the ball state at impacts, tracks (well-posed) reference trajectories with arbitrary precision. Additionally, the trajectory tracking control strategy is not limited to plants with a single juggling object; it also solves the problem of stabilizing multiple balls to different rhythmic patterns.

To the best of our knowledge, there are no previous results in the literature that solve the (multiple-object) trajectory tracking problem for juggling systems. Additionally, the modeling and control design techniques we introduce in this paper can be extended to more general mechanical systems with impacts, including applications ranging from control of walking and jumping robots to robotic manipulators and beyond.

\section{HYBRID MODELING OF JUGGLING SYSTEMS}

\section{A. General model and solution concept}

We consider juggling systems with plant given by

$$
\dot{x}_{1}=f_{1}\left(x_{1}\right),
$$

where $x_{1}=\left[\begin{array}{ll}x_{11}^{\top} & x_{12}^{\top}\end{array}\right]^{\top} \in \mathbb{R}^{n_{1}}$ is the state, and actuated robot given by

$$
\dot{x}_{2}=f_{2}\left(x_{2}, u\right),
$$

where $x_{2}=\left[\begin{array}{ll}x_{21}^{\top} & x_{22}^{\top}\end{array}\right]^{\top} \in \mathbb{R}^{n_{2}}$ is the state and $u \in \mathbb{R}^{m}$ is the control input. The components $x_{11}$ and $x_{21}$ of the states $x_{1}$ and $x_{2}$ correspond to the position state, while the components $x_{12}$ and $x_{22}$ correspond to the velocity state of the plant and actuated robot, respectively. Let $x:=\left[\begin{array}{ll}x_{1}^{\top} & x_{2}^{\top}\end{array}\right]^{\top}$ and $f(x, u):=\left[f_{1}\left(x_{1}\right)^{\top} f_{2}\left(x_{2}, u\right)^{\top}\right]^{\top}$.

The impact law between the plant and the actuated robot is given by the difference equations

$$
x_{1}^{+}=g_{1}(x), \quad x_{2}^{+}=g_{2}(x),
$$

where $x_{1}^{+}, x_{2}^{+}$denote the value of the state $x_{1}, x_{2}$ after the impact. Let $g(x):=\left[g_{1}(x)^{\top} g_{2}(x)^{\top}\right]^{\top}$. The impacts between the plant and the actuated robot are assumed to occur when, for a continuously differentiable function $h: \mathbb{R}^{n_{1}+n_{2}} \rightarrow \mathbb{R}$, the state $x$ and the input $u$ satisfy

$$
h(x)=0 \text { and }\langle\nabla h(x), f(x, u)\rangle \leq 0 .
$$


We interpret the juggling system above as the hybrid system

$$
\begin{aligned}
\dot{x} & =f(x, u) & & h(x) \geq 0, \\
x^{+} & =g(x) & & h(x)=0 \text { and }\langle\nabla h(x), f(x, u)\rangle \leq 0,(6)
\end{aligned}
$$

where $f$ and the state constraint in (5) define the flow map and flow set, respectively, and $g$ and the state constraints in (6) define the jump map and jump set, respectively. We follow the framework for hybrid systems in [4], [5] where solutions are given on hybrid time domains by hybrid arcs. A set $E$ is a hybrid time domain if for all $(T, J) \in E$, $E \cap([0, T] \times\{0,1, \ldots J\})$ is a compact hybrid time domain, i.e. it can be written as

$$
\bigcup_{j=0}^{J-1}\left(\left[t_{j}, t_{j+1}\right], j\right)
$$

for some finite sequence of times $0=t_{0} \leq t_{1} \ldots \leq t_{J}$. A hybrid arc $x$ is a function defined on a hybrid time domain $\operatorname{dom} x$ mapping to a state space such that $x(t, j)$ is locally absolutely continuous in $t$ for each $j,(t, j) \in \operatorname{dom} x$. The notation used in (5)-(6) is suggestive of the meaning of solutions. Vaguely, for an input $u: \operatorname{dom} u \rightarrow \mathbb{R}^{m}$, a hybrid arc $x: \operatorname{dom} x \rightarrow \mathbb{R}^{n}$ is a solution to a hybrid system (5)-(6) if $\operatorname{dom} x=\operatorname{dom} u$ and $x(t, j)$ satisfies: (C1) $\dot{x}(t, j)=f(x(t, j), u(t, j))$ and $h(x(t, j)) \geq 0$ for almost all $t$ in every nontrivial interval $\left[t_{j}, t_{j+1}\right],(t, j) \in \operatorname{dom} x$, and (C2) $x^{+}(t, j):=x(t, j+1)=g(x(t, j)), h(x(t, j))=0$, and $\langle\nabla h(x(t, j)), f(x(t, j), u(t, j))\rangle \leq 0$ for all $(t, j)$ such that $(t, j),(t, j+1) \in \operatorname{dom} x$. For more details, see [4], [5].

\section{B. One degree-of-freedom juggler}

For the one degree-of-freedom juggler depicted in Figure 1, the dynamics of the ball (plant) are given by Newton

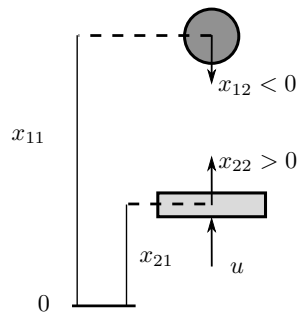

Fig. 1. One degree-of-freedom juggler: one ball (plant) and actuated robot. Their positions are denoted by $x_{11}, x_{21}$ and their velocities by $x_{12}, x_{22}$, respectively.

laws

$$
\dot{x}_{1}=\left[\begin{array}{c}
x_{12} \\
-\gamma
\end{array}\right]=: f_{1}\left(x_{1}\right),
$$

where $x_{1}:=\left[\begin{array}{ll}x_{11} & x_{12}\end{array}\right]^{\top} \in \mathbb{R}^{2}, x_{11}$ is the height, $x_{12}$ is the velocity of the ball, and $\gamma$ is the gravity constant. The mass of the plant is denoted by $m_{1}$. The actuated robot is assumed to have double integrator dynamics given by

$$
\dot{x}_{2}=\left[\begin{array}{c}
x_{22} \\
u
\end{array}\right]=: f_{2}\left(x_{2}, u\right),
$$

where $x_{2}:=\left[\begin{array}{ll}x_{21} & x_{22}\end{array}\right]^{\top} \in \mathbb{R}^{2}, x_{21}$ is the height, $x_{22}$ is the velocity of the actuated robot, and $u \in \mathbb{R}$ is the control input. The mass of the actuated robot is denoted by $m_{2}$.

Impacts are modeled by an impact rule with conservation of momentum [1], [13]:

$$
\begin{aligned}
x_{12}^{+}-x_{22}^{+} & =-e\left(x_{12}-x_{22}\right), \\
m_{1} x_{12}^{+}+m_{2} x_{22}^{+} & =m_{1} x_{12}+m_{2} x_{22},
\end{aligned}
$$

where $e \in(0,1)$ is the restitution coefficient. Let $\lambda=$ $\frac{m_{1}}{m_{1}+m_{2}}$. Then, the update law at impacts velocities is

$$
\begin{aligned}
{\left[\begin{array}{l}
x_{12}^{+} \\
x_{22}^{+}
\end{array}\right] } & =\left[\begin{array}{cc}
\lambda-(1-\lambda) e & (1-\lambda)(1+e) \\
\lambda(1+e) & 1-\lambda-\lambda e
\end{array}\right]\left[\begin{array}{l}
x_{12} \\
x_{22}
\end{array}\right] \\
& =: \Gamma(\lambda, e)\left[\begin{array}{l}
x_{12} \\
x_{22}
\end{array}\right]
\end{aligned}
$$

while the update law for positions is given by

$$
x_{11}^{+}=x_{11}, \quad x_{21}^{+}=x_{21} .
$$

The impacts between the ball and the actuated robot occur when $x_{11}=x_{21}$ and $x_{12} \leq x_{22}$.

Then, the one degree-of-freedom juggler system in Figure 1 is given by the hybrid system $\mathcal{H}$ with flows

$$
\left.\begin{array}{l}
\dot{x}_{11}=x_{12}, \quad \dot{x}_{12}=-\gamma \\
\dot{x}_{21}=x_{22}, \quad \dot{x}_{22}=u
\end{array}\right\} \quad x_{11}-x_{21} \geq 0,
$$

and jumps

$$
\left.\begin{array}{rl}
x_{11}^{+} & =x_{11} \\
x_{12}^{+} & =\left[\begin{array}{ll}
1 & 0
\end{array}\right] \Gamma(\lambda, e)\left[\begin{array}{l}
x_{12} \\
x_{22}
\end{array}\right] \\
x_{21}^{+} & =x_{21} \\
x_{22}^{+} & =\left[\begin{array}{ll}
0 & 1
\end{array}\right] \Gamma(\lambda, e)\left[\begin{array}{c}
x_{12} \\
x_{22}
\end{array}\right]
\end{array}\right\} \begin{gathered}
x_{11}-x_{21}=0 \\
\text { and } x_{12}-x_{22} \leq 0 .
\end{gathered}
$$

Note that for this system, the flow and jump sets, denoted by $C$ and $D$, respectively, can be defined as in (5)-(6) with $h(x):=x_{11}-x_{21}$ (the condition $\langle\nabla h(x), f(x, u)\rangle \leq 0$ becomes $\left.x_{12}-x_{22} \leq 0\right)$ :

$$
\begin{aligned}
C & :=\left\{x \in \mathbb{R}^{4} \mid x_{11}-x_{21} \geq 0\right\}, \\
D & :=\left\{x \in \mathbb{R}^{4} \mid x_{11}-x_{21}=0, x_{12}-x_{22} \leq 0\right\} .
\end{aligned}
$$

\section{Trajectory Tracking With Hybrid CONTROL}

In this section, we introduce a trajectory tracking problem for the one degree-of-freedom juggler in Section II-B and propose a hybrid control strategy that solves it. The main ideas and concepts are applicable when solving trajectory tracking problems for more general classes of mechanical systems with impacts.

\section{A. Reference trajectories for tracking}

We generate rhythmic juggling patterns with the hybrid system $\mathcal{H}_{r}$ given by

$$
\begin{array}{ll}
\dot{r}_{1}=r_{2}, \quad \dot{r}_{2}=-\gamma & r_{1}-r_{1}^{*} \geq 0, \\
r_{1}^{+}=r_{1}, \quad r_{2}^{+}=-r_{2} & r_{1}-r_{1}^{*}=0 \text { and } r_{2} \leq 0,
\end{array}
$$

where $r_{1}^{*}$ is the reference height parameter. Let $r:=\left[r_{1} r_{2}\right]^{\top}$. Given an initial condition $r^{0}, r_{1}^{0} \geq r_{1}^{*}$, the solution $r$ to $\mathcal{H}_{r}$ 
defines a reference trajectory for tracking on a hybrid time domain $\operatorname{dom} r$. The reference $r$ has impacts at $r_{1}=r_{1}^{*}$; velocity after the impact given by minus the velocity before the impact, where the velocity value after the impact defines the reference velocity parameter $r_{2}^{*}$; and impact period given by $T_{r}=2 r_{2}^{*} / \gamma$. To guarantee that the reference trajectories are "well-posed" (in the sense that they can be tracked by $\mathcal{H}$ ), the flow map of $\mathcal{H}_{r}$ is given by (7). Figure 2 depicts a reference trajectory $r$ with $r_{1}^{*}, r_{2}^{*}>0$.

\section{B. Finite-time $\varepsilon$-tracking}

We are interested in practical tracking of the ball position given a reference signal $r$ generated by $\mathcal{H}_{r}$. Both $x_{1}$ and $r$ are given on hybrid time domains which do not necessarily need to be the same. By tracking between $x_{1}$ and $r$ we mean that their graphs are close after a finite amount of time.

Definition 3.1 (finite-time $\varepsilon$-tracking): Given $\varepsilon \geq 0$ and hybrid $\operatorname{arcs} x_{1}: \operatorname{dom} x_{1} \rightarrow \mathbb{R}^{2}, r: \operatorname{dom} r \rightarrow \mathbb{R}^{2}, x_{1}$ and $r$ are $\varepsilon$-close after $T \geq 0$ if

(a) for all $(t, j) \in \operatorname{dom} x_{1}$ with $(t, j) \succeq(T, J)$ for some $J$, $(T, J) \in \operatorname{dom} x_{1}$, there exists $\left(t^{\prime}, j^{\prime}\right) \in \operatorname{dom} r,\left|t-t^{\prime}\right| \leq$ $\varepsilon$, and

$$
\left|x_{1}(t, j)-r\left(t^{\prime}, j^{\prime}\right)\right| \leq \varepsilon,
$$

(b) for all $(t, j) \in \operatorname{dom} r$ with $(t, j) \succeq(T, J)$ for some $J$, $(T, J) \in \operatorname{dom} r$, there exists $\left(t^{\prime}, j^{\prime}\right) \in \operatorname{dom} x_{1},\left|t-t^{\prime}\right| \leq$ $\varepsilon$, and

$$
\left|r(t, j)-x_{1}\left(t^{\prime}, j^{\prime}\right)\right| \leq \varepsilon .
$$

When this property holds for $x_{1}$ and a given reference trajectory $r$, we will call it finite-time $\varepsilon$-tracking, and we will say that " $x_{1}$ finite-time $\varepsilon$-tracks $r$ ". With an appropriate offset of $x_{1}$ and $r$, finite-time $\varepsilon$-tracking corresponds to the notion of graphical closeness of solutions to hybrid systems introduced in [4] (see also [5]).

\section{Problem statement and control strategy}

We want to solve the following control problem:

( $\star$ Given a level of tracking accuracy $\varepsilon>0$ and a reference trajectory $r$ generated by $\mathcal{H}_{r}$, the ball state component $x_{1}$ of the solutions to $\mathcal{H}$ finite-time $\varepsilon$-tracks the reference trajectory $r$ with only measurements of $x_{1}$ at impact times.

To solve it, we propose the following control algorithm (see Figure 2):

- Algorithm for Single-ball Juggling: At every impact between the ball and the actuated robot (say, it occurs at hybrid time $\left(t_{0}, 0\right)$ and that after the jump, the hybrid time is $\left.\left(t_{0}, 1\right)\right)$ :

Step 1) Compute the apex time of the trajectory describing the ball position $\left(x_{11}\right)$ resulting from the impact (denote this time by $t_{a}$ );

Step 2) Solve for the time of the next two consecutive impacts after $t_{a}$ in the reference signal $r$. Denote these impact times by $t_{1}^{\prime}$ and $t_{2}^{\prime}$, respectively. If $t_{1}^{\prime}=t_{a}$ then define $\left(t_{1}, t_{2}\right)$ to be either $\left(t_{1}^{\prime}, t_{2}^{\prime}\right)$ or $\left(t_{1}^{\prime}+T_{r}, t_{2}^{\prime}+T_{r}\right)$. Otherwise, $\left(t_{1}, t_{2}\right)=\left(t_{1}^{\prime}, t_{2}^{\prime}\right)$.
Step 3) Compute the ball trajectory $x_{1}$ at $\left(t_{1}, 1\right)$ (thus, assuming no impacts between time $t_{0}$ and $t_{1}$ );

Step 4) Compute the value of the state $x_{2}$ at $\left(t_{1}, 1\right)$, denoted by $x_{2}^{\prime}$, required for the value of $x_{1}$ after the impact at $\left(t_{2}, 2\right)$, that is, $x_{1}\left(t_{2}, 3\right)$, to be equal to the reference trajectory $r$;

Step 5) Generate a virtual reference trajectory $z$ that at time $\left(t_{1}, 1\right)$ is equal to the value of $x_{2}$, given by $x_{2}^{\prime}$, computed in Step 4).

Finally, the control law applied to the actuated robot is designed so that $x_{2}$ tracks the virtual reference trajectory computed in Step 5).

Note that Steps 1)-5) can be computed by explicitly solving the dynamics of $\mathcal{H}$. The virtual reference trajectory in Step 5), denoted by $z$, is a trajectory that satisfies the dynamics of the actuated robot. At the impact time, it is reset to a value that guarantees that, when tracked by the actuated robot, the next impact occurs at the appropriate time (at this time instant, $x_{2}$ is equal to $x_{2}^{\prime}$ ). Figure 2

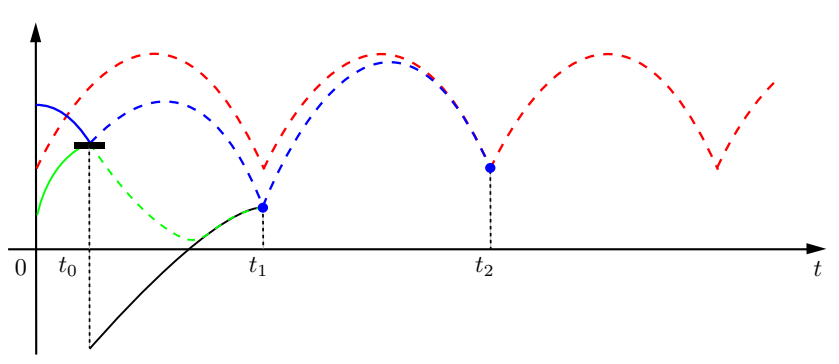

Fig. 2. Main control idea to track a reference trajectory $r$ ( $r_{1}$ component plot in red, dashed). At the impact at $t=t_{0}$, the controller computes the resulting ball position trajectory $x_{11}$ (blue, dashed) at time $t_{1}$ and the required value of the state $x_{2}$ at $t_{1}$ such that the next desired impact time $t_{2}$ of the reference, $x_{1}$ equals $r$. The virtual reference trajectory $z$ (black) resulting from this computation is tracked by the actuated robot (green, dashed).

depicts the computations in Steps 1)-5) that the control algorithm performs at the impact at $\left(t_{0}, 0\right)$. For simplicity, the trajectories are plotted projected to the ordinary time axis $t$ of their hybrid time domain.

\section{Hybrid controller}

We implement the control algorithm above in a hybrid controller, which we denote by $\mathcal{H}_{c}$. Its state is given by $z=$ $\left[\begin{array}{ll}z_{1} & z_{2}\end{array}\right]^{\top} \in \mathbb{R}^{2}$, the virtual reference state. The controller performs three main tasks:

- At every impact, perform computations in Step 1)-4).

- At every impact, reset $z$ to a value such that the continuous dynamics of $z$ generate a virtual reference trajectory that matches the impact constraint in Step 4).

- In between impacts, control the actuated robot to track the virtual reference trajectory $r$.

We define the continuous dynamics of the state $z$ by a copy of the dynamics of the actuated robot. Then, the flows of $\mathcal{H}_{c}$ are given by

$$
\dot{z}_{1}=z_{2}, \quad \dot{z}_{2}=\alpha
$$


where $\alpha<0$. This constant is chosen so that the $z_{1}$ components of the solution to (10) are described by concave parabolas (see [9] for a rigorous robustness analysis regarding the selection of such parameter). The jump map for $\mathcal{H}_{c}$ is given by

$$
\left[\begin{array}{l}
z_{1} \\
z_{2}
\end{array}\right]^{+} \in \kappa_{c}\left(x_{1}, z, r\right)
$$

where $\kappa_{c}: \mathbb{R}^{2} \times \mathbb{R}^{2} \times \mathbb{R}^{2} \rightrightarrows \mathbb{R}^{2}$ is a set-valued mapping, as it will become clear in the next section, that updates the state $z$ for the generation of the virtual trajectory. The output of the controller is given by

$$
u=\kappa\left(x_{2}, z\right)
$$

where $u$ is the control input to the actuated robot and $\kappa: \mathbb{R}^{2} \times$ $\mathbb{R}^{2} \rightarrow \mathbb{R}^{2}$. As (11) suggests, the hybrid controller $\mathcal{H}_{c}$ uses only the states $x_{1}, z$ and reference information at impacts for the update of $z$.

The closed-loop system resulting from controlling the juggling system $\mathcal{H}$ with the hybrid controller $\mathcal{H}_{c}$ can be written as the following hybrid system, which we denote by $\mathcal{H}_{c l}$, with state space $O:=\mathbb{R}^{6}$ :

$$
\begin{aligned}
& \left.\begin{array}{l}
\dot{x}_{11}=x_{12}, \quad \dot{x}_{12}=-\gamma \\
\dot{x}_{21}=x_{22}, \quad \dot{x}_{22}=\kappa\left(x_{2}, z\right) \\
\dot{z}_{1}=z_{2}, \quad \dot{z}_{2}=\alpha
\end{array}\right\} x_{11}-x_{21} \geq 0, \\
& x_{11}^{+}=x_{11} \\
& x_{12}^{+}=\left[\begin{array}{ll}
1 & 0
\end{array}\right] \Gamma(\lambda, e)\left[\begin{array}{l}
x_{12} \\
x_{22}
\end{array}\right] \\
& x_{21}^{+}=x_{21} \\
& x_{22}^{+}=\text {[0 } \\
& {\left[\begin{array}{c}
z_{1} \\
z_{2}
\end{array}\right]^{+} \in \kappa_{c}\left(x_{1}, z, r\right)} \\
& x_{11}-x_{21}=0 \\
& \text { and } \\
& x_{12}-x_{22} \leq 0 .
\end{aligned}
$$

\section{E. Control design and main results}

To design the update law $\kappa_{c}$ of the hybrid controller $\mathcal{H}_{c}$, we initially replace the dynamics of the actuated robot in $\mathcal{H}$ by the dynamics of the state $z$ in $\mathcal{H}_{c}$. That is, we consider the hybrid system

$$
\begin{aligned}
& \left.\begin{array}{l}
\dot{x}_{11}=x_{12}, \quad \dot{x}_{12}=-\gamma \\
\dot{z}_{1}=z_{2}, \quad \dot{z}_{2}=\alpha
\end{array}\right\} x_{11}-z_{1} \geq 0, \\
& \left.\begin{array}{rl}
x_{11}^{+} & =x_{11} \\
x_{12}^{+} & =\left[\begin{array}{ll}
1 & 0
\end{array}\right] \Gamma(\lambda, e)\left[\begin{array}{c}
x_{12} \\
z_{2}
\end{array}\right] \\
{\left[\begin{array}{l}
z_{1} \\
z_{2}
\end{array}\right]^{+}} & \in \kappa_{c}\left(x_{1}, z, r\right)
\end{array}\right\} \begin{array}{c}
x_{11}-z_{1}=0 \\
\text { and } \\
x_{12}-z_{2} \leq 0 .
\end{array} .
\end{aligned}
$$

We denote this system by $\mathcal{H}_{v}$ meaning virtual juggling system. The control design idea is to define the set-valued map $\kappa_{c}$ such that the control task $(\star)$ is accomplished for $\mathcal{H}_{v}$ and then design the control law $\kappa$, which acts on the actuated robot, to accomplish asymptotic tracking between $x_{2}$ and $z$ during flows. To that end, we first state the following result for the solutions to $\mathcal{H}_{v}$. Below, by feasible initial condition of $\mathcal{H}_{v}$ we mean any initial condition for which solutions to $\mathcal{H}_{v}$ never reach $x_{11}=z_{1}, x_{12}=z_{2}$.

Lemma 3.2: For every feasible initial condition $\left[\begin{array}{llll}x_{11}^{0} & x_{12}^{0} & z_{1}^{0} & z_{2}^{0}\end{array}\right]^{\top}$ of $\mathcal{H}_{v}$, the next impact occurs at time $\left(t_{1}, 0\right)$, where $t_{1}$ is given by the nonnegative solution to

$$
z_{1}^{0}=-\frac{\gamma+\alpha}{2} t_{1}^{2}+\left(x_{12}^{0}-z_{2}^{0}\right) t_{1}+x_{11}^{0} .
$$

Moreover, the position and velocity of the ball after the impact at $\left(t_{1}, 0\right)$, denoted by $x_{11}\left(t_{1}, 1\right)$ and $x_{12}\left(t_{1}, 1\right)$, respectively, are given by

$$
\begin{aligned}
x_{11}\left(t_{1}, 1\right) & =-\frac{\gamma}{2} t_{1}^{2}+x_{12}^{0} t_{1}+x_{11}^{0}, \\
x_{12}\left(t_{1}, 1\right) & =\left[\begin{array}{ll}
1 & 0
\end{array}\right] \Gamma(\lambda, e)\left[\begin{array}{c}
x_{12}^{0}-\gamma t_{1} \\
\alpha t_{1}+z_{2}^{0}
\end{array}\right] .
\end{aligned}
$$

Lemma 3.2 can be shown by solving explicitly for $x_{1}$ and $z$. In fact, (12) follows from solving the system backward in time from the jump condition of $\mathcal{H}_{v}$, (13) follows since at jumps, the $x_{11}$ component of the solution is mapped to itself, and (14) is derived from the impact rule in $\mathcal{H}_{v}$.

Let $J: \mathbb{R}^{2} \times \mathbb{R}^{2} \times \mathbb{R}^{2} \rightrightarrows \mathbb{R}^{2}$ be the set-valued mapping

$$
\begin{aligned}
& J\left(x_{1}, z, r\right):= \\
& \left\{\begin{array}{cl}
\frac{r_{2}+r_{2}^{*}}{\gamma} & \text { if } \frac{a x_{12}+b z_{2}}{\gamma}<\frac{r_{2}+r_{2}^{*}}{\gamma} \\
\left.\frac{r_{2}+r_{2}^{*}}{\gamma}, \frac{r_{2}+r_{2}^{*}}{\gamma}+T_{r}\right\} & \text { if } \frac{a x_{12}+b z_{2}}{\gamma}=\frac{r_{2}+r_{2}^{*}}{\gamma} \\
\frac{r_{2}+r_{2}^{*}}{\gamma}+T_{r} & \text { if } \frac{a x_{12}+b z_{2}}{\gamma}>\frac{r_{2}+r_{2}^{*}}{\gamma}, \\
a=\left[\begin{array}{ll}
1 & 0
\end{array}\right] \Gamma(\lambda, e)\left[\begin{array}{l}
1 \\
0
\end{array}\right], \quad b=\left[\begin{array}{ll}
1 & 0
\end{array}\right] \Gamma(\lambda, e)\left[\begin{array}{l}
0 \\
1
\end{array}\right] .
\end{array}\right.
\end{aligned}
$$

Our control algorithm first computes the time for the next impact $t_{1}$ in Step 1) and then computes Step 2)-5) to generate a virtual trajectory. Regarding Step 1), the setvalued mapping $J$ defines the time(s) to the next impact, given by $t_{1}$, from the current state. If the apex time of the trajectory $x_{11}$ is smaller than the time for the next impact of the reference, then $t_{1}$ is given by the next impact time of the reference. If, instead, the apex time of the trajectory $x_{11}$ is larger than the time for the next impact of the reference, then the impact is postponed for one period $T_{r}$. When $t_{1}$ is equal to the apex time, both times are possible and, therefore, $J$ is set valued. Regarding Step 2)-5), for each $t_{1} \in J\left(x_{1}, z, r\right)$, the reset value $z^{*}$ for $z$ is computed by two applications of Lemma 3.2. We do this by setting $x_{1}\left(t_{2}, 3\right)=r^{*}, t_{1}+T_{r}$ (see Figure 2). Then, the set-valued mapping $\kappa_{c}$ is given for each $x_{1}, z, r \in \mathbb{R}^{2}$ by all points $z^{*}=\left[\begin{array}{ll}z_{1}^{*} & z_{2}^{*}\end{array}\right]^{\top}$ satisfying

$$
\begin{aligned}
& z_{1}^{*} \in-\frac{\gamma+\alpha}{2} \tilde{t}^{2}+\left(a x_{12}+b z_{2}-z_{2}^{*}\right) \tilde{t}+x_{11} \\
& z_{2}^{*} \in \frac{r_{1}^{*}+\frac{\gamma}{2} T_{r}^{2}+\frac{\gamma}{2} \tilde{t}^{2}-\left(a x_{12}+b z_{2}\right) \tilde{t}-x_{11}}{b T_{r}} \\
&+\frac{(a \gamma-b \alpha) \tilde{t}-a\left(a x_{12}+b z_{2}\right)}{b} \\
& \text { for each } \tilde{t} \in J\left(x_{1}, z, r\right) .
\end{aligned}
$$

The control law $\kappa$ is designed so that the trajectories of the actuated robot system track the virtual reference trajectories. 
In a perfect tracking scenario, when the error between the actuated robot state and the virtual trajectory is zero, the control algorithm achieves finite-time 0 -tracking. This is actually the case for the virtual juggling system $\mathcal{H}_{v}$.

Theorem 3.3: For each reference trajectory $r$ generated from $\mathcal{H}_{r}$ and each feasible initial condition of $\mathcal{H}_{v}$, each solution to $\mathcal{H}_{v}$ is bounded and the $x_{1}$ component finite-time 0 -tracks the reference trajectory $r$. Moreover, the trajectories coincide after three impacts.

In general, there is an error between $x_{2}$ and $z$. Let $e_{1}:=$ $x_{21}-z_{1}, e_{2}:=x_{22}-z_{2}$. Then, the error system is

$$
\dot{e}_{1}=e_{2}, \quad \dot{e}_{2}=u-\alpha
$$

Given $k_{1}, k_{2}>0$, a particular choice of the control law $\kappa$ to accomplish the tracking between $x_{2}$ and $z$ is given by

$$
\kappa\left(x_{2}, z\right)=\alpha-k_{1}\left(x_{21}-z_{1}\right)-k_{2}\left(x_{22}-z_{2}\right) .
$$

We now state the main result of this section. As defined for $\mathcal{H}_{v}$, feasible initial conditions for $\mathcal{H}_{c l}$ correspond to initial conditions from which solutions to $\mathcal{H}_{c l}$ never reach the condition $x_{11}=x_{21}, x_{12}=x_{22}$.

Theorem 3.4: For each compact set $K \subset O$, each $\varepsilon>0$, and each reference trajectory $r$ generated from $\mathcal{H}_{r}$, there exists $k_{1}, k_{2} \in \mathbb{R}$ such that each solution to $\mathcal{H}_{c l}$ starting from $K$ that is feasible is bounded and the $x_{1}$ component finite-time $\varepsilon$-tracks the reference trajectory $r$. Moreover, only three impacts are required for $x_{1}$ and $r$ to be $\varepsilon$-close.

Remark 3.5: The proof of Theorem 3.3 follows from the construction of the update law $\kappa_{c}$, which is designed so that the ball component of solutions to $\mathcal{H}_{v}$ converge to the reference trajectory in finite time. To show Theorem 3.4, we establish that, on compact sets, the error between the nominal trajectories and the trajectories with perturbed impact time (by a mismatch between $x_{2}$ and $z$ ) vanishes with the mismatch between $x_{2}$ and $z$. Then, the desired tracking precision given by $\varepsilon$ can be obtained by choosing fast enough converging tracking law $\kappa$ so that at the impact times, the state of the actuated robot is within appropriate level of perturbation. This condition is satisfied by selecting large enough parameters $k_{1}, k_{2}$ of the tracking law $\kappa$. Using this same proof technique, we are also able to show that the closed-loop system is robust to measurement noise, computation errors, and observer-based output feedback. Due to space constraints, we do not pursue this here.

\section{F. Simulations}

We simulate the closed-loop system $\mathcal{H}_{c l}$ with a reference trajectory generated by $\mathcal{H}_{r}$ with $r_{1}^{*}=0 \mathrm{~m}, r_{2}^{*}=10 \mathrm{~m} / \mathrm{s}$, and initial condition $r^{0}=[0 \mathrm{~m} 10 \mathrm{~m} / \mathrm{s}]^{\top}$.

Figure 3 shows a simulation of the closed-loop system. For simplicity, we present the trajectories projected to the ordinary time $t$ axis. The ball trajectory approaches the reference trajectory in the neighborhood of the time corresponding to the third bounce. Note that the parameters of the control law $\kappa$ steer the actuated robot to a very small neighborhood of

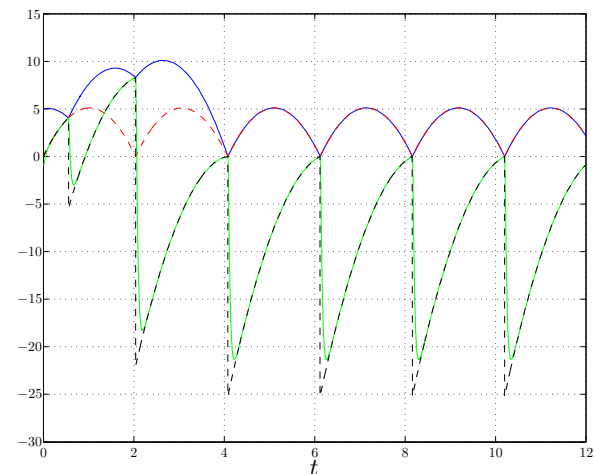

Fig. 3. Simulation of closed-loop system $\mathcal{H}_{c l}$. System parameters: $m_{1}=$ $1 \mathrm{Kg}, m_{2}=9 \mathrm{Kg}, e=0.8, \gamma=9.8 \mathrm{~m} / \mathrm{s}^{2}$. Controller parameters: $\alpha=-9.8, k_{1}=2000, k_{2}=100$. Initial condition: $x_{11}(0,0)=5 \mathrm{~m}$, $x_{12}(0,0)=1 \mathrm{~m} / \mathrm{s}, x_{21}(0,0)=-1 \mathrm{~m}, x_{22}(0,0)=0 \mathrm{~m} / \mathrm{s}$. The trajectory of the ball (blue) impacts with the actuated robot (trajectory in green). Finitetime $\varepsilon$-tracking is achieved at the third bounce when the ball trajectory approaches the reference trajectory (red, dashed). The virtual reference $z$ is depicted with black, dashed line.

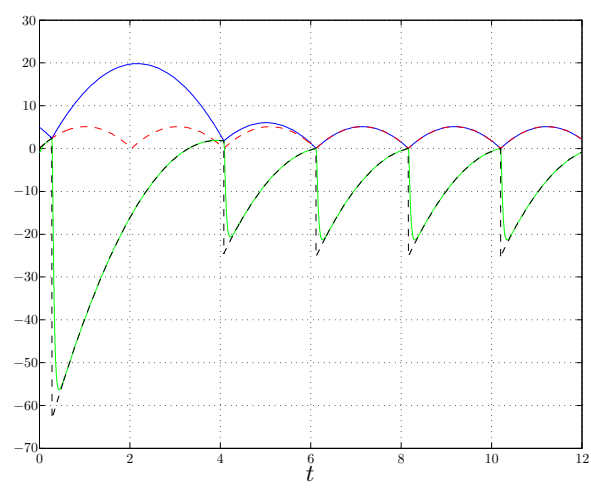

Fig. 4. Simulation of closed-loop system $\mathcal{H}_{c l}$. System parameters: $m_{1}=$ $1 \mathrm{Kg}, m_{2}=9 \mathrm{Kg}, e=0.8, \gamma=9.8 \mathrm{~m} / \mathrm{s}^{2}$. Controller parameters: $\alpha=-9.8, k_{1}=2000, k_{2}=100$. Initial condition: $x_{11}(0,0)=5 \mathrm{~m}$, $x_{12}(0,0)=-8 \mathrm{~m} / \mathrm{s}, x_{21}(0,0)=-1 \mathrm{~m}, x_{22}(0,0)=0 \mathrm{~m} / \mathrm{s}$. The trajectory of the ball (blue) impacts with the actuated robot (trajectory in green). Finite-time $\varepsilon$-tracking is achieved at the third bounce when the ball trajectory approaches the reference trajectory (red, dashed). The virtual reference $z$ is depicted with black, dashed line.

the virtual reference. This level of closeness can be made arbitrarily small by choosing large enough $k_{1}$ and $k_{2}$.

The simulation in Figure 4 is for the same reference trajectory but for different initial conditions of the ball. It illustrates the decision that the controller makes when the apex time of the trajectory after the first bounce is larger than the next impact of the reference trajectory. As a difference to the simulation in Figure 3, the second impact is planned for $t_{1}=4 r_{2}^{*} / \gamma$ rather than for $t_{1}=2 r_{2}^{*} / \gamma$.

\section{The Multiple-Balls Juggling Case}

In this section, we consider the multiple ball juggling problem. Suppose we are given $n$ reference trajectories, $n$ balls, and one actuated robot. Our goal is the following:

( $\star$ ) Given a level of tracking accuracy $\varepsilon>0$ and $n$ reference trajectories generated by $\mathcal{H}_{r}$ with distinct impact times, the $i$-th ball state component $x_{1}^{i}$ of the solutions to 
the closed-loop system finite-time $\varepsilon$-tracks the $i$-th reference trajectory $r^{i}$ with only measurements of $x_{1}^{i}$ at impact times.

We propose a strategy that combines the control algorithm introduced in Section III to plan the impacts for each ball individually and uses additional logic to select the ball to control. Let $Q:=\{1,2, \ldots, n\}$ and $q$ be a logic state, $q \in$ $Q$. Let $z^{q} \in \mathbb{R}^{2}$ be the virtual reference state of the $q$-th ball. The reference trajectory for $q$-th ball is generated by the hybrid system $\mathcal{H}_{r}^{q}$. We assume that for each $q, \mathcal{H}_{r}^{q}$ is defined as $\mathcal{H}_{r}$. For problem $(\star \star)$, we further assume that the reference trajectories are such that the impact times do not occur at the same time and that they have the above ordering property: every $n$ impacts, each reference trajectory has only one impact, and the order is preserved. The control logic for multiple-ball juggling is as follows.

- Algorithm for Multiple-ball Juggling: At an impact between the $q$-th ball and the actuated robot:

Step 1) With reference trajectory $r^{q}$, compute Step 1)-5) of the Algorithm for Single-ball Juggling to obtain $z^{q *}$. Update the state $z^{q}$ with this value.

Step 2) Update the logic state $q$ by $q^{+}=\bmod (q, n)+1$.

Step 3) Apply to the actuated robot a control law that tracks the virtual reference $z^{q}$.

We implement this logic in a hybrid controller and obtain the closed-loop system $\mathcal{H}_{c l}^{M}$ given by

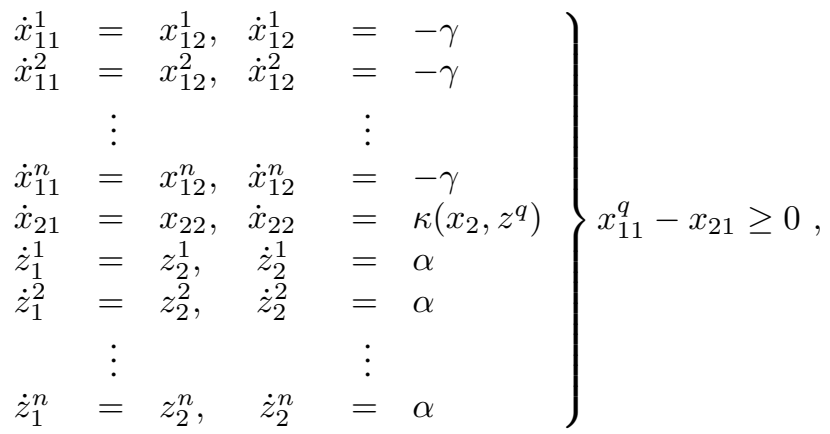

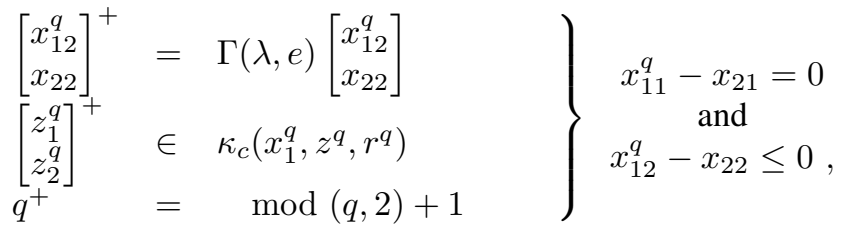

where in the jump map and jump set, we have omitted the states that remain constant during flows and jumps.

By construction, the closed-loop system $\mathcal{H}_{c l}^{M}$ inherits the same properties than the ones of $\mathcal{H}_{c l}$ in Theorem 3.4. The main difference in the multiple trajectory tracking problem is that feasible initial conditions need to satisfy more restrictive constraints: every $n$ impacts, each ball has impacted only once, and the order is preserved.

We will just mention that the controller construction for multiple (and consequently, for the single-ball case) trajectory tracking is such that the conditions for nominal robustness of hybrid systems in [4] and [5] hold.
Figure 5 shows simulations results for three-balls juggling. The reference trajectories have a $120 \mathrm{deg}$ phase difference between each other. The plots show that each ball $\varepsilon$-tracks the corresponding reference trajectory after the third impact.

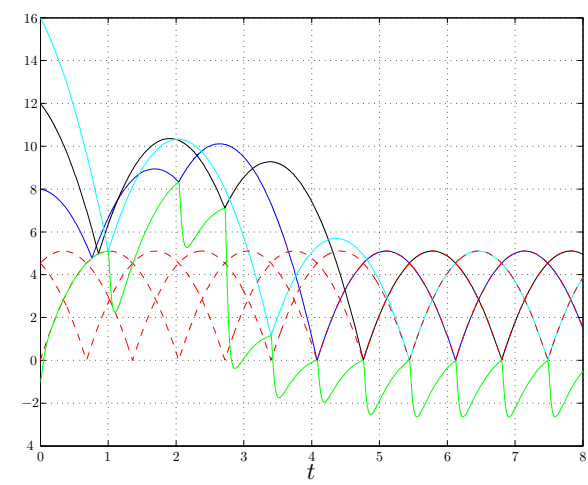

Fig. 5. Simulation of the closed-loop system $\mathcal{H}_{r}^{M}$ for three balls. System parameters: $m_{1}^{1}=m_{1}^{2}=m_{1}^{3}=1 \mathrm{Kg}, m_{2}=9 \mathrm{Kg}, e=0.8, \gamma=$ $9.8 \mathrm{~m} / \mathrm{s}^{2}$. Controller parameters: $\alpha=-9.8, k_{1}=2000, k_{2}=100$. For the given initial conditions, the trajectories approach their respective reference trajectories (in red, dashed) at their third bounce.

\section{REFERENCES}

[1] B. Brogliato. Nonsmooth Mechanics Models, Dynamics and Control. Springer, 1996.

[2] B. Brogliato and A. Zavala-Rio. On the control of complementaryslackness juggling mechanical systems. IEEE Trans. Aut. Cont., 45(2):235-246, Feb. 2000.

[3] M. Buehler, D.E. Koditschek, and P.J. Kindlmann. Planning and control of robotic juggling and catching tasks. The International Journal of Robotics Research, 13(2):101-118, 1994.

[4] R. Goebel, J.P. Hespanha, A.R. Teel, C. Cai, and R.G. Sanfelice. Hybrid systems: Generalized solutions and robust stability. In Proc. 6th IFAC Symposium in Nonlinear Control Systems, pages 1-12, 2004.

[5] R. Goebel and A.R. Teel. Solutions to hybrid inclusions via set and graphical convergence with stability theory applications. Automatica, 42(4):573-587, 2006.

[6] K. M. Lynch and C.K. Black. Recurrence, controllability, and stabilization of juggling. IEEE Transactions on Robotics and Automation, 17(2):113-124, 2001.

[7] J.J. Moreau. Topics in Nonsmooth Mechanics, chapter Bounded variation in time, pages 1-74. Birkhäuser Verlag, 1988.

[8] R. Sepulchre R. Ronsse, P. Lefevre. Sensorless stabilization of bounce juggling. IEEE Transactions on Robotics, 22(1):147-59, 2006.

[9] R. Ronsse, P. Lefèvre, and R. Sepulchre. Rhythmic feedback control of a blind planar juggler. IEEE Transactions on Robotics, 2007.

[10] R.G. Sanfelice, R. Goebel, and A.R. Teel. A feedback control motivation for generalized solutions to hybrid systems. In J. P. Hespanha and A. Tiwari, editors, Hybrid Systems: Computation and Control: 9th International Workshop, volume LNCS 3927, pages 522536, 2006.

[11] S. Schaal and C. G. Atkeson. Open loop stable control strategies for robot juggling. In IEEE International Conference on Robotics and Automation, volume 3, pages 913-918, 1993.

[12] R. Sepulchre and M. Gerard. Stabilization of periodic orbits in a wedge billiard. In Proc. 42nd IEEE Conference on Decision and Control, pages 1568-1573, 2003.

[13] A. van der Schaft and H. Schumacher. An Introduction to Hybrid Dynamical Systems. Lecture Notes in Control and Information Sciences, Springer, 2000.

[14] T.L. Vincent. Controlling a ball to bounce at a fixed height. In Proceedings of the American Control Conference, volume 1, pages 842-846, 1995.

[15] A. Zavala-Rio and B. Brogliato. On the control of a one degree-offreedom juggling robot. Dynamics and Control, 9:67-90, 1999. 